\title{
NONLINEAR LASER AMPLIFIER WITH SUPPRESSED LEVEL OF QUANTUM NOISE ON THE BASIS OF A BOSE CONDENSATE FOR ${ }^{23}$ Na ATOMS
}

\author{
A. V. Prokhorov, A. P. Alodjants, A. Yu. Leksin, S. M. Arakelian \\ Vladimir State University ${ }^{1}$, Vladimir, Russia
}

\begin{abstract}
Based on advanced quantum theory for the $\Lambda$-scheme nonlinear interaction of optical fields in atomic Bose-Einstein condensate, the regimes of giant negative nonlinearity and absorption coefficients have been obtained for a probe light pulse. The nonlinear laser amplifier setup (under the $\Lambda$-scheme realization) for generation of the quadrature-squeezed light is presented.

На основе квантовой теории для схемы нелинейного взаимодействия оптических полей в атомном бозе-эйнштейновском конденсате показана возможность реализации режима с гигантскими отрицательными значениями нелинейных показателя преломления и коэффициента поглощения пробного импульса света. Предложена схема нелинейного лазерного усилителя (на основе схемы взаимодействия) с возможностью генерации квадратурно-сжатых состояний света.
\end{abstract}

\section{INTRODUCTION}

The physical effects such as electromagnetically induced transparency [1], laser generation without inversion [2], the giant increase of the refractive index in resonant medium [3] are the basic directions in modern quantum and nuclear optics for a long time. But new opportunities for experimental realization of such coherent effects are discovered by nuclear systems, including atomic Bose-Einstein condensates (BEC) [4]. The fundamental aspect of the phenomena is determined by quantum interference for the effects of light absorption and reemission in multilevel nuclear systems [5]. The nonlinear approach to the problem is a practical perspective when optical media with high-efficient nonlinear interactions are used. The fact results in realization of multiparametric dispersive relations in such a system [6], and, e.g., the suppression of fluctuations for output light is developed [7]. As a result, a very well known regime of the noiseless laser amplification effect [8] for the case can be used to open the new opportunities for nonlinear control of the signal-noise ratio in a probe beam propagation process.

In the present work we consider a resonant coherent nonlinear interaction for light in a Bose-Einstein condensate of sodium atoms under conditions of electromagnetically induced light amplification in $\Lambda$-scheme. We obtained for the first time a principal possibility of the quadrature-squeezed light generation due to amplification effect for a probe field.

${ }^{1}$ E-mail: laser@vpti.vladimir.ru 


\section{THE ANALYSIS OF NONLINEAR OPTICAL PROCESSES IN A $\Lambda$-SCHEME INTERACTION}

We assume that the BEC of ${ }^{23} \mathrm{Na}$ atoms (atomic levels $|1\rangle,|2\rangle,|3\rangle$ ) is irradiated by two optical fields (Fig. 1), i.e., the high intensity pump field $\mathbf{E}_{c}(z, t)$ under condition of a narrow resonance for transition $|1\rangle \rightarrow|3\rangle$ (the pump pulse is of long duration) and the weak and shorter probe pulse $\mathbf{E}_{p}(z, t)$ with the central frequency $\omega_{p}$ being resonance-off (the detuning parameter $\Delta$ ) from the transition $|2\rangle \rightarrow|3\rangle$.

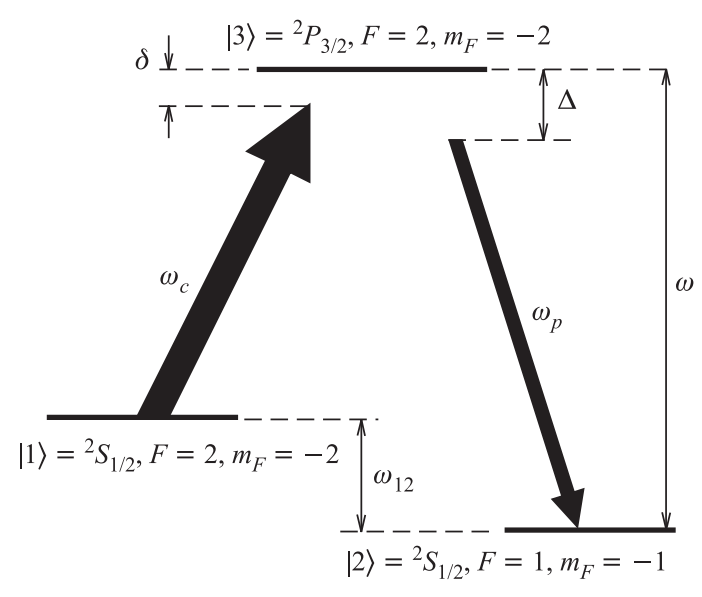

Fig. 1. $\Lambda$-scheme of interaction for ${ }^{23} \mathrm{Na}$ atomic energy levels

For considered $\Lambda$-scheme we are using a standard density-matrix approach [9]. The coupled system of equations for the density-matrix elements $\bar{\rho}_{m n}$ can be obtained by very well known means of Liouville equation [10] and described many times (see, e.g., [7]).

Taking into account nonlinear effects, the matrix element $\bar{\rho}_{32}$ can be expanded in a series on the Rabi frequency for a probe field $g_{2}$ in the form

$$
\bar{\rho}_{32}=\bar{\rho}_{32}^{(0)}+\bar{\rho}_{32}^{(1)} g_{2}+\bar{\rho}_{32}^{(2)}\left|g_{2}\right|^{2}+\bar{\rho}_{32}^{(3)}\left|g_{2}\right|^{2} g_{2},
$$

where $\bar{\rho}_{32}^{(0)}=0$ determines the spontaneous polarization of atoms (absent for ${ }^{23} \mathrm{Na}$ atoms), $\bar{\rho}_{32}^{(1)}$ represents the linear solution, $\bar{\rho}_{32}^{(2)}$ and $\bar{\rho}_{32}^{(3)}$ are the nonlinear corrections for nonlinearity of second order $\chi_{A T}^{(2)}$ (equivalent to zero for symmetric molecules [11]) and for cubic nonlinearity $\chi_{A T}^{(3)}$, correspondingly.

Expression (1) is true for two cases: when the Rabi frequency $g_{2}$ is negligible in comparison with the frequency detuning of a probe pulse and/or when the $g_{2}$ frequency is negligible in comparison with the decay time inverse value of optical level (the resonance), i.e., when $g_{2}<\Delta$ and/or $g_{2}<\gamma_{32}$ (in a resonance) [12].

The optical properties of the BEC described by nonlinear polarization of atoms in the form (1) can be presented in the terms of the refractive index $n=n_{0}+n_{2}\left|A_{p}\right|^{2}$ and 
absorption coefficient $\alpha=\alpha_{0}+\alpha_{2}\left|A_{p}\right|^{2}$. Here, $A_{p}$ is the field amplitude (maximal value of the light pulse),

$$
n_{0}=1+\frac{1}{2} \operatorname{Re}\left(\chi_{A T}^{(1)}\right), \alpha_{0}=\beta_{p} \operatorname{Im}\left(\chi_{A T}^{(1)}\right), n_{2}=\frac{3}{8} \operatorname{Re}\left(\chi_{A T}^{(3)}\right), \alpha_{2}=\frac{3}{4} \beta_{p} \operatorname{Im}\left(\chi_{A T}^{(3)}\right) .
$$

Using the microscopic approach for definition of polarization in the medium [10], we obtain the following expressions for resonant susceptibilities (cf. with [6]):

$$
\chi_{A T}^{(1)}=\frac{N\left|\mu_{32}\right|^{2}}{\hbar \varepsilon_{0}} \frac{1}{\Gamma}, \quad \chi_{A T}^{(3)}=\frac{2 i}{3} \frac{N\left|\mu_{32}\right|^{4}}{\varepsilon_{0} \hbar^{3}} \frac{\Gamma^{*}-\Gamma}{\Gamma|\Gamma|^{2}}\left(\frac{1}{\gamma_{\mathrm{opt}}}+\frac{1}{\gamma_{\mathrm{mag}}}\right),
$$

where $N$ is the atomic density; $\mu_{32}$ is the corresponding dipole momentum; $\Gamma=\Delta-i \gamma_{\text {opt }}+$ $\left|g_{1}\right|^{2} /\left(i \gamma_{\mathrm{mag}}-\Delta\right) ; \gamma_{\mathrm{opt}}=\gamma_{32}+\gamma_{31}, \gamma_{\mathrm{mag}}=\gamma_{12}, g_{1}$ is the Rabi frequency of a pump beam.

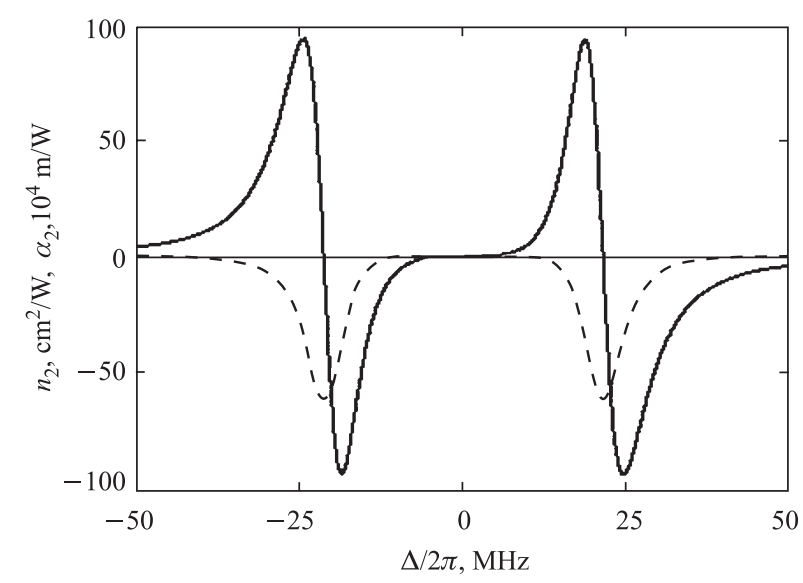

Fig. 2. Frequency dependences of the nonlinear refractive index $n_{2}$ (solid line) and nonlinear absorption coefficient $\alpha_{2}$ (dashed line) for the ${ }^{23} \mathrm{Na}$ condensate upon the $\Lambda$-interaction with external optical fields. The condensate parameters are $N=3.3 \cdot 10^{12} \mathrm{~cm}^{-3}, \gamma_{\mathrm{opt}} / 2 \pi=10.2 \mathrm{MHz}, \gamma_{\mathrm{mag}} / 2 \pi=38.2 \mathrm{kHz}$. The intensity of a transparency pulse is equal to $I_{c}=55 \mathrm{~mW} / \mathrm{cm}^{2}$

Figure 2 shows the nonlinear refractive index $n_{2}$ and the nonlinear absorption coefficient $\alpha_{2}$ as functions of the probe field frequency detuning $\Delta$ for the BEC ${ }^{23} \mathrm{Na}$ atoms density $N=3.3 \cdot 10^{12} \mathrm{~cm}^{-3}$ and for the pump beam intensity $I_{c}=55 \mathrm{~mW} / \mathrm{cm}^{2}$. One can see from Fig. 2 that parameters $n_{2}$ and $\alpha_{2}$, induced by a pump beam, reach a very high value and can be negative. In the case $\alpha_{2}<0$ the effective amplification for a probe field intensity is observed due to nonlinear interaction with the excited BEC. The fact corresponds to electromagnetically induced amplification of light. Simultaneously, the conditions for negative cubic nonlinearity evidence are determined which is of practical significance in problems of nonclassical light generation [13]. However, observation in experiment of considered regimes is under question due to probe field linear absorption and the medium dispersive effects development. But to overcome the problem we have to choose the necessary intensity of a probe field $I_{p}$ and the frequency detuning $\Delta$ suitable for realization of the compensation regime for two opposite effects, i.e., the linear absorption and nonlinear amplification of a probe field. Other possibility 
is realized by compensation of a second-order dispersion $k_{2}=\partial^{2} k / \partial \omega^{2}$ at the chosen point (marked $A$ ) on the dispersive curve.

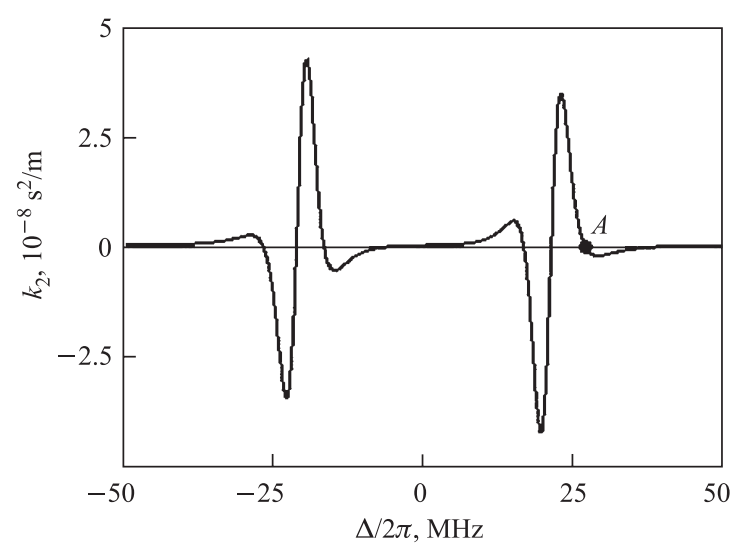

Fig. 3. Frequency dependences of the group velocity dispersion $k_{2}$ for the probe pulse in ${ }^{23} \mathrm{Na}$ condensate upon the $\Lambda$-interaction with external optical fields. The parameters of the system are the same as for Fig. 2. The intensity of a probe pulse is equal to $I_{p}=5.5 \mathrm{~mW} / \mathrm{cm}^{2}$

Figure 3 shows the parameter $k_{2}$ as a function of frequency detuning $\Delta$ for a probe field. For duration $\tau_{p} \approx 1 \mu \mathrm{s}$ and intensity $I_{p}=5.5 \mathrm{~mW} / \mathrm{cm}^{2}$ the point $A$ in Fig. 3 corresponds to discussed effect of the dispersion compensation. At this point $n_{2}=-74 \mathrm{~cm}^{2} / \mathrm{W}$ and the high level of amplification for a probe field is observed. We will discuss the regime later.

\section{NONLINEAR AMPLIFICATION AND GENERATION OF THE QUADRATURE-SQUEEZED LIGHT}

We shall consider in this section the quantum properties of a probe field for the light amplification regime in BEC. By using the mathematical apparatus of secondary quantization, the process of nonlinear interaction of a quantum probe field and the coherent atomic medium (i.e., BEC) can be presented by the following Hamiltonian [13]:

$$
H_{\mathrm{int}}=-\frac{k_{0}^{2}}{2}\left(\bar{\rho}_{32}^{(1)} a^{\dagger}+\bar{\rho}_{23}^{(1)} a\right)-\frac{k_{0}^{4}}{2}\left(\bar{\rho}_{32}^{(3)} a^{\dagger} N_{f}+\bar{\rho}_{23}^{(3)} N_{f} a\right)
$$

where $a\left(a^{\dagger}\right)$ is the annihilation (creation) operator for photons in probe field; $N_{f}=a^{\dagger} a$ is the operator of photons number; $k_{0}=\mu_{32} \sqrt{\omega /\left(2 \hbar \varepsilon_{0} V\right)} ; V$ is the interaction volume. The first term in Eq. (4) corresponds to the interaction of the BEC with the probe field in the framework of the Jaynes-Cummings model [14]. The second term in Eq. (4) is determined by nonlinear polarization of atomic system. This approximation will be carried out in the case of adiabatically slow switched probe field in the presence of pump beam on condition that $g_{1} \gg \gamma_{32}$ [15]. In addition, the time delay value of a probe pulse in the BEC should be shorter than a typical lifetime of the ground levels; i.e., $\tau_{d} \leqslant \tau_{12}$ [16], where $\tau_{d}=n_{g} l / c$, $n_{g}=n+\omega_{p}\left(d n / d \omega_{p}\right)$ is a group refractive index. In this case the density matrix elements of the system achieve quickly stationary values and do not depend on time. 
To describe the behavior of quantum fluctuations for a probe field, we are using the Bogoliubov method [17], according to which the photon annihilation operator of a probe field can be represented in the form $\hat{a}=f+\hat{c}$, where $f=\langle\hat{a}\rangle$ determines the classical amplitude and the operator $\hat{c}=\hat{a}-\langle\hat{a}\rangle$ determines the small fluctuation part of the field. Using Eq. (3) in the Heisenberg representation, we arrive at the following system of equations for the mean field and quantum-noise operators [13]:

$$
\frac{d f}{d t}=i\left(\frac{k_{1}}{2}+\frac{k_{2}^{*}}{2} f^{2}+k_{2}|f|^{2}\right) ; \quad \frac{d \hat{c}}{d t}=i\left(\theta \cdot \hat{c}+\eta \hat{c}^{\dagger}\right),
$$

where $k_{1}=k_{0}^{2} \rho_{32}^{(1)}$ and $k_{2}=k_{0}^{4} \rho_{32}^{(3)}$ are the coefficients characterizing the linear and nonlinear energy repumping in the system, correspondingly; $\theta=k_{2} f^{*}+k_{2}^{*} f$ and $\eta=k_{2} f$.

It can be shown for the point $A$ in Fig. 3 that in linear domain a weak absorption of a probe field is observed. But for the time scale of about $5 \mathrm{~ns}$ the intensity of a probe field remains almost the initial value. At the same time scale in the nonlinear interaction case the parametrical increasing of photons number is observed for a probe pulse.

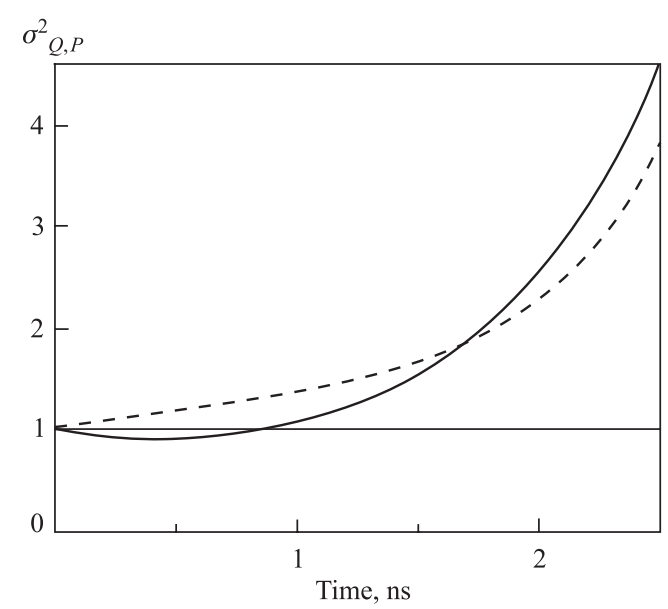

Fig. 4. Time dependences for quadrature dispersions $\sigma_{P}^{2}$ (solid line) and $\sigma_{Q}^{2}$ (dashed line). Value $\sigma_{Q, P}^{2}=1$ corresponds to coherent noise level at the input. The parameters of the system correspond to nonlinear regime

Let us determine the Hermitian quadratures $Q=a+a^{\dagger}$ and $P=i\left(a^{\dagger}-a\right)$ of the probe field. Figure 4 shows the time dependences for the variations $\sigma_{Q}^{2}$ and $\sigma_{P}^{2}$ of two quadratures in amplification regime. By analogy with the case of linear phase-sensitive amplifier, enhancement of noise in the $P$ quadrature is delayed by faster enhancement of noise in the $Q$ quadrature. However, for nonlinear probe field amplification the noise of the $P$ quadrature can fall below the standard quantum limit $\left(\sigma_{Q, P}^{2}=1\right.$ in Fig. 4$)$, which is impossible in linear amplification. Thus, under nonlinear amplification of a probe field the noise level for one of the quadratures may be suppressed, even to negative values, which may result in generation of nonclassical quadrature-squeezed light (in BEC for the considered case). The high-level suppression of quantum fluctuations may be practically observed at 
the characteristic time scale $\tau_{\mathrm{sq}} \approx 0.5 \mathrm{~ns}$. So, due to the analysis carried out, a nonlinear laser amplifier can work under certain conditions as a nonclassical light generator, i.e., as a generator of amplitude-squeezed light [7].

\section{CONCLUSION}

We presented for the first time the nonlinear regimes of propagation of optical pulses in a BEC of three-level atoms for the $\Lambda$-scheme of interaction. The setup of nonlinear laser amplifier which can generate a quadrature-squeezed light is discussed. The process is caused by the high efficiency of nonlinear optical transformations for a probe field in the BEC in the presence of intensive pump field when the conditions of negative absorption and nonlinearity are realized.

Acknowledgements. This work was supported by the Russian Foundation for Basic Research, project No.04-02-17359, and by the Ministry of Education and Science of the Russian Federation.

\section{REFERENCES}

1. Harris S. E., Field J. E., Imamoglu A. // Phys. Rev. Lett. 1990. V. 64. P. 1107.

2. Kocharovskaya O.A., Khanin Ya.I. // Pis'ma ZhETF. 1988. V.48. P. 581.

3. Fleischhauer M. et al. // Opt. Commun. 1992. V. 87. P. 109.

4. Anderson M. H. et al. // Science. 1995. V. 269. P. 198.

5. Arimondo E., Orriols G. // Nuovo Cim. Lett. 1976. V. 17. P. 333.

6. Wang H., Goorskey D., Xiao M. // Phys. Rev. Lett. 2001. V. 87. P. 073601.

7. Vadeiko I. et al. // Phys. Rev. A. 2005. V.72. P. 013804.

8. Scully M. O., Zubairy M. S. // Opt. Commun. 1988. V.66. P. 303.

9. Vasiliev N. A., Troshin A. S. // Izv. RAN. Ser. Fiz. 2004. V.68. P. 1277.

10. Shen Y.R. The Principles of Nonlinear Optics. Berkeley: Univ. of California, 1984.

11. Akhmanov S.A., Vysloukh V.A., Chirkin A.S. Optics of Femtosecond Laser Pulses. M.: Nauka, 1988.

12. Klyshko D. N. Physical Foundations of Quantum Electronics. M.: Nauka, 1986.

13. Prokhorov A. V., Alodjants A. P., Arakelian S. M. // JETP Lett. 2004. V.80. P. 870.

14. Jaynes E. T., Cummings F. W. // Proc. IEEE. 1963. V.51. P. 89.

15. Rathe U. et al. // Phys. Rev. A. 1993. V.47. P. 4994.

16. Lukin M. D. // Rev. Mod. Phys. 2003. V.75. P. 457.

17. Lifshitz E. M., Pitaevsky L.P. Statistical Physics. M.: Nauka, 1978. V.2. 